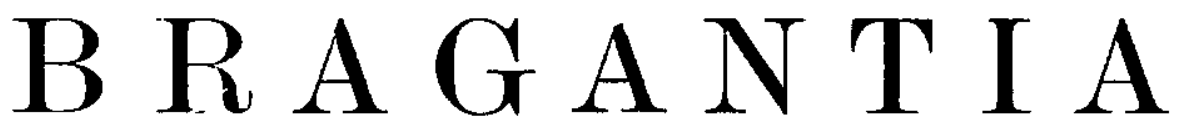

Revista Cientifica do Instituto Agronômico do Estado de Säo Paulo

\title{
FINEZA E COMPRIMENTO DE FIBRA DE DOZE VARIEDADES DE RAMI ( $\left.{ }^{1}\right)$
}

Gullherme Augusto de Paiva Castro, Seção de Plantas Fibrosas. Instituto Agronômico

\section{SINOPSE}

Estudaram-se a fineza e o comprimento de fibra de doze variedades interracionais de rami (Boehmeria nivea Gaud.) pertencentes à coleção existente no Instituto Agronômico do Estado de Săo Paulo.

A variedade Taiwan Hakushi destacou-se como produtora de fibras mais tinas, enquanto a Hsi-Ching-Chin-Hsin foi a que apresertou fibras mais grossas.

Com relaçāo ao comprimento de fibra, as variedades P.I. 87.521, Banmethuot e Taiwan Hakushi tiveram os valores mais baixos, apresentando porcentagens mais elevadas de fibras curtas, e Hakuhi lbgou, Hsi-Ching-Chin-Hsin e Shalien n. ${ }^{\circ} 1$ apresentaram comprimentos médios de fibras mais altos, destacando-se por porcentagens mais elevadas de fibras longas.

\section{1 - INTRODUÇÃO}

A tibra de rami vem sendo utilizada na sua maior quantidade para fabricaçāo de fios destinados à confecção de variados tipos de tecido. A fineza e o comprimento da fibra têm grande importância para a indústria têxtil, pois é, principalmente, dessas características que irá depender o título do fio fabricado e, por conseguinte, o tipo do tecido que se pode confeccionar. Será, portanto, de grande interesse conhecer esses valores para as diversas variedades de rami, devido à preferência que os industriais dão às fibras de natureza mais fina e de bom comprimento médio.

A fineza da fibra é expressa em número métrico ( $\mathrm{Nm}$ ), que é o número de quilômetros encontrado em um quilograma de fibra. Nos

(1) Trabatho apresentado no $\mathrm{V}$ Congresso Nacional de Técnicos Téxteis, realizado em Recife, Estado de Pernambuco, de 7 a 10 de setembro de 1972, Recebido para

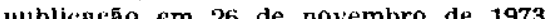


trabalhos existentes sobre a fineza de fibra do rami, são encontrados valores diferentes para essa característica, os quais podem ser devidos às variedades ou aos métodos utilizados. Roehrich e Nhuân (9) determinaram, para a variedade Angola, $\mathrm{Nm}=1855$. Vreeswijk (11) cita que, no Japão, para atender às exigências dos industriais, existenı em cultivo variedades de fibras finas, apesar de apresentarem menor produção de fibras por área. Castro e Correia (2) obtiveram $\mathrm{Nm}=1724$, para a variedade de rami Murakami, utilizando as fibras extraídas de todo o comprimento do caule. Grillot e Franquim (4) determinaram $\mathrm{Nm}=3703$, sem especificar a variedade. Medina, Ciaramello, Toselo e Venturini ( $\boldsymbol{\gamma})$ encontraram valores diferentes de fineza da fibra de rami com relação às épocas de colheita, posição das fibras nos caules e irrigação. Seale e Allison (8) relatam que a fineza depende da variedade; finalmente, Ciaramello, Medina e Salgado (3) constataram que a fineza das fibras de rami está relacionada com o diâmetro dos caules e que o comprimento dos caules não tem qualquer influência sobre a fineza das fibras.

Com referência ao comprimento de fibra, Beauverie (1), Kirby (5), Mathews (6), Tonelli (10) e Roehrich \& Nhuân (9) dão valores que variam de 60 a 500 milímetros, não especificando as variedades utilizadas. Castro e Correia ( $\boldsymbol{z}$ ) determinaram para a variedade Murakami um comprimento médio de 200 milimetros, utilizando um método diferente do empregado neste trabalho.

No presente estudo, são apresentados os resultados obtidos sobre a fineza e o comprimento das fibras de doze variedades de rami, assim como uma distribuição das fibras em diversas classes de comprimento.

\section{2 - MATERIAL E MÉTODOS}

Em uma experiência em blocos ao acaso, com quatro repetições, instalada no Centro Experimental de Campinas, em Latossolo Roxo, estudaram-se a fineza e o comprimento de fibra de doze variedades de rami, provenientes de diversas regiōes do mundo. Essas variedades são relacionadas a seguir. 


\begin{tabular}{|c|c|c|c|}
\hline VARJEDADE & $\begin{array}{l}\text { Forma de } \\
\text { obtençâo }\end{array}$ & Origem & $\begin{array}{c}\text { Número de } \\
\text { introdução (2) }\end{array}$ \\
\hline Banmethuot & Semente & Est. Unidos & 20.527 \\
\hline E $47-1 \quad \ldots \ldots \ldots \ldots \ldots \ldots \ldots \ldots \ldots \ldots$ & Semente & Est. Unidos & 20.528 \\
\hline Hakuhi Ibgou $\quad \ldots \ldots \ldots \ldots \ldots \ldots$ & Semente & Japăo & 22.385 \\
\hline Hsi-Ching-Chin-Hsin $\quad \ldots \ldots \ldots \ldots$ & Semente & Formosa & 31.165 \\
\hline Kagisei $\quad \ldots \ldots \ldots \ldots \ldots \ldots \ldots$ & Sernente & Japão & 22.388 \\
\hline Miyasaki $112 \quad \ldots \ldots \ldots \ldots \ldots \ldots$ & Semente & Formosa & 17.713 \\
\hline Murakami $\ldots$ & Rizoma & Brasil & 17.660 \\
\hline P.I. $87.521 \quad \ldots \ldots \ldots \ldots \ldots \ldots \ldots$ & Rizoma & Est. Unidos & 31.166 \\
\hline Shalien n. $^{\circ} 1 \quad \ldots \ldots \ldots \ldots \ldots \ldots$ & Semente & Formosa & 17.714 \\
\hline Taiwan Hakushi $\ldots \ldots \ldots \ldots \ldots$ & Rizoma & Formosa & 13.488 \\
\hline White Skin $\quad \ldots \ldots \ldots \ldots \ldots \ldots$ & Semente & Formosa & 31.167 \\
\hline Yilan $n^{*} \quad 1 \quad \ldots \ldots \ldots \ldots \ldots \ldots$ & Semente & Formosa & 31.164 \\
\hline
\end{tabular}

Com a finalidade de obter amostras homogêneas colheram-se, dos canteiros de cada repetição, cinco caules bem uniformes quanto ao diâmetro e estado de maturação. As plantas foram cortadas em 7-1-1970, data considerada como a do início da brotação dos novos caules.

Quando os novos caules atingiram a maturidade, foram colhidos a pequenos intervalos de dias, devido à necessidade de trabalhar grande número de amostras. Assim, os caules das variedades White Skin, Hsi-Ching-Chin-Hsin e P.I. 87.521 foram colhidos com 89 dias após o início da brotação; os da variedade Murakami, Banmethuot e Miyasaki 112, con 91 dias de idade; os das variedades Kagisei, Yilan n. ${ }^{\circ} 1$ e Hakuhi Ibgou, com 92 dias; e os das variedades E 47-1, Taiwan Hakushi e Shalien n. 1 com 93 dias de idade.

O método utilizado na obtenção das amostras de fibras foi baseado no trabalho de Grillot e Franquim (4), com algumas modificações, com a finalidade de adaptá-lo às condições de trabalho disponíveis.

Após a colhcita, os caules foram desfolhados a mão e medidos os seus comprimentos por meio de uma escala graduada em milímetros. Marcado o meio do caule, de cada um deles tirou-se um pedaço de 250 milímetros de comprimento, com 125 milímetros acima e 125 milimetros abaixo do ponto marcado. Esses pedaços, em número de cinco para cada canteiro, foram pesados, etiquetados e tratados em uma solução de $\mathrm{NaOH}$ a $1 \%$ durante uma hora e meia, sob fervura.

(2) Registro na Seçáo de Botånłca Economica, Lab. de Introd. de Plantas. 
A seguir, sob a ação de um filete de água, a simples pressão dos dedos sobre a superfície dos pedaços de caules foi suficiente para provocar a eliminação da película.

Com a retirada da película, procedeu-se à separação das fibras propriamente ditas da parte lenhosa restante. Os feixes de fibras obtidos de cada pedaço foram secos em estufa à temperatura de $100-110^{\circ} \mathrm{C}$, até peso constante, e, posteriormente, amaciados e submetidos a uma leve penteagem. De cada feixe, retiraram-se vinte fibras inteiras, que foram medidas individualmente, por meio de uma escala graduada em milimetros. Depois de medidas, cada conjunto de vinte fibras, correspondente a cada pedaço de caule, foi pesado em balança elétrica de precisão. Dividindo o resultado da soma dos comprimentos das vinte fibras pelo peso do seu conjunto obteve-se um número que é o índice de fíneza da fibra, expresso em número métrico $(\mathrm{Nm})$. Como muitos autores adotam o denier (peso em gramas de $9000 \mathrm{me}-$ tros de fibra) como índice de fineza da fibra, ele é também apresentado no presente trabalho, obtido pela simples transformação dos valores de número métrico.

As fibras depois de medidas foram agrupadas em três classes de comprimento: curta (abaixo de $143 \mathrm{~mm}$ ), média (entre 143 e $179 \mathrm{~mm}$ ) e longa (acima de $179 \mathrm{~mm}$ ). Os limites dessas classes foram de caráter pessoal do autor, com a finalidade de determinar se as variedades possuiam tendências de produzir quantidades diferentes de fibras curtas, médias ou longas, dando uma indicação da uniformidade de fibra de cada variedade.

\section{3 - RESUltados E discussáo}

\section{1 - FINEZA DAS FIBRAS}

No quadro 1 estão indicados os valores médios obtidos, em número métrico e denier, da fineza das fibras para as doze variedades de rami.

Oc resultados da análise da variância efetuada com os valores de número métrico revelaram uma diferença altamente significativa entre as variedades.

Comparando as médias das varicdades pelo teste de Tukey, ao nível de $5 \%$ de probabilidade, verificou-se que a fineza média de 


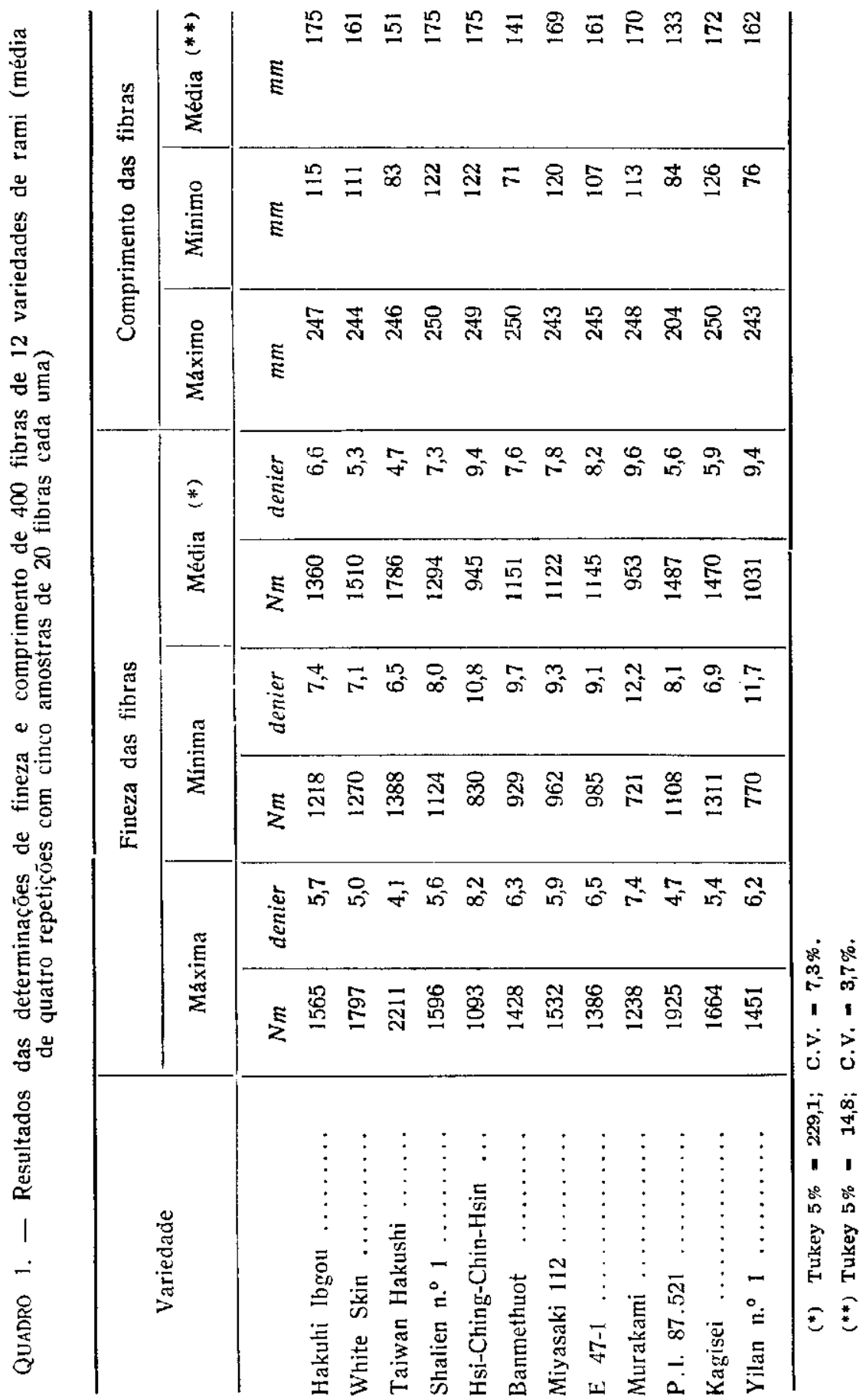


fibra da variedade Taiwan Hakushi foi superior à das demais, com um índice médio de fineza em $\mathrm{Nm}=1786$. As variedades White Skin, P.I. 87.521 e Kagisei, com números métricos respectivamente de 1510,1487 e 1470 , apresentaram índices médios de fineza superiores ao das variedades Banmethuot, E-47-1, Miyasaki 112, Yilan $n .{ }^{\circ} 1$, Murakami e Hsi-Ching-Chin-Hsin, cujos valores foram, em ordem, de 1151, 1145, 1122, 1031, 953 e 945.

A variedade Hakuhi lbgou, com $\mathrm{Nm}=1360$, foi supiror às variedades Miyasaki 112, Yilan n. ${ }^{\circ}$, Murakami e Hsi-Ching-Chin-Hsin. Finalmente, a Shalien $n .^{\circ} 1$, com $\mathrm{Nm}=1294$, foi superior às variedades Yilan n. ${ }^{\circ} 1$, Murakami e Hsi-Ching-Chin-Hsin, grupo esse que apresentou os menores índices médios de fineza de fibra.

O coeficiente de variabilidade foi de $7,3 \%$.

\section{2 - COMPRIMENTO DAS FIBRAS}

Os resultados médios do comprimento de fibra, em milímetros, estāo indicados no quadro 1. A análise da variância dos valores obtidos revelou que houve diferença altamente significativa entre as variedades.

O teste de Tukey, ao nível de $5 \%$ de probabilidade, mostrou que as variedades Banmethuot e P.I. 87.521, com comprimentos médios de fibras de 141 e 133 milimetros, respectivamente, foram inferiores às demais, à exceção da variedade Taiwan Hakushi. Entre Shalien n. ${ }^{\circ}$, Hakuhi Ibgou, Hsi-Ching-Chin-Hsin, Kagisei, Murakami 112, Yilan n. 1 , E 47-1 e White Skin não houve diferenças significativas. Seus comprimentos médios de fibras variaram de 161 milimetros, para as variedades White Skin e E 47-1, a 175 milimetros, para Hakuhi Ibgou, Hsi-ChingChin-Hsin e Shalien $n^{\circ} 1$. Taiwan Hakushi, com um comprimento médio de 151 milimetros, mostrou-se significativamente superior a P.I. 87.521 e inferior a Shalien n. ${ }^{\circ} 1$, Taiwan Hakushi, Hsi-ChingChin-Hsin, Kagisei, Murakami e Miyasaki 112.

O coeficiente de variabilidade foi bastante baixo, com um valor de $3,7 \%$, considerado muito bom para essa espécie de experimento.

Nas condições do presente estudo, o comprimento máximo de fibra esperado seria de 250 milímetros, correspondente ao comprimento dos pedaços de caules utilizados na amostragem. Diversos autores (1, $6, \mathbf{9}, \mathbf{1 0}$ ) obtiveram valores superiores a esse comprimento, alcançando 
comprimentos médios de fibra mais elevados. Essas diferenças podem ser devidas, principalmente, aos métodos empregados nas determinações e às variedades estudadas.

\subsection{1 - CLASSES DE COMPRIMENTO DE FIBRAS}

Com a finalidade de conhecer a porcentagem de fibras curtas, médias e longas, e a uniformidade de fibra de cada variedade de rami, as quatrocentas fibras de cada uma foram agrupadas em classes de comprimento.

Os dados do quadro 2 mostram que as variedades P.I. 87.521, Banmethuot e Taiwan Hakushi apresentaram proporções elevadas de fibras curtas, respectivamente com $68,8 \%, 58,8 \%$ e $42,3 \%$. As variedades Hakuhi lbgou, Hsi-Ching-Chin-Hsin e Shalien n. 1 destacaram-se como produtoras de fibras longas, correspondendo aos valores de $42,5 \%, 41,5 \%$ e $38,0 \%$. Em relação à proporção de fibras médias, sobressairant-se as seguintes variedades, com mais de $50 \%$ de fibras classificadas nesse grupo: Kagisei $(55,8 \%)$, E $47-1 \quad(53,0 \%)$ e Shalien $n .^{\circ} 1(50,5 \%)$.

$\mathrm{Na}$ figura 1 estão representados os histogramas de freqüência, em número e porcentagem, das quatrocentas fibras de cada classe de comprimento, para as variedades de rami.

Um exame da referida figura revela que as variedades de comprimento médio de fibra mais elevado tiveram uma menor amplitude de dispersão dos elemntos em torno da média, o que indica maior uniformidade do comprimento de fibra. Nas variedades de baixo comprimento médio de fibra, houve maior amplitude de dispersăo, resultando em menor uniformidade dessa característica.

As características estudadas de fineza, comprimento e uniformidade de fibra são de grande importância para a indústria têxtil, pois delas depende a técnica a ser empregada para realizar uma boa fiação da fibra. 


\begin{tabular}{|c|c|c|c|c|c|c|c|c|c|c|c|c|c|c|c|c|}
\hline 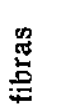 & \multicolumn{2}{|c|}{ 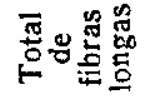 } & 20 & $\stackrel{12}{9}$ & ญै & $\approx$ & $\overbrace{}^{\circ}$ & 7 & & है & & $\begin{array}{l}\text { ָे } \\
\text { พิ }\end{array}$ & हे & ${ }_{10}^{\infty}$ & m5 & ले \\
\hline $\begin{array}{l}\frac{4}{0} \\
\frac{n}{\pi}\end{array}$ & \multirow{4}{*}{ 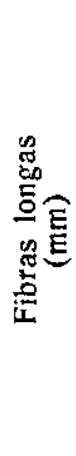 } & 芯 & 30 & 12 & $\stackrel{\infty}{0}^{\infty}$ & 10 & $\stackrel{N}{*}$ & ni & $0_{0}^{\infty}$ & 7 & $=$ & $\stackrel{0}{=}$ & ni & 1 & in & iv \\
\hline$\sum_{0}^{n}$ & & $\begin{array}{l}\text { लु } \\
\text { ஸे } \\
\text { ஸे }\end{array}$ & 80 & $\stackrel{\infty}{m}$ & in & 5 & $\stackrel{\infty}{\nabla^{\prime}}$ & $\stackrel{0}{\infty}$ & $\stackrel{N}{2}$ & 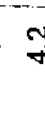 & 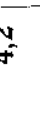 & $\stackrel{v}{i}$ & $F$ & 1 & $\stackrel{\infty}{m}$ & $\stackrel{v}{m^{\prime}}$ \\
\hline $\begin{array}{l}\overline{\mathscr{L}} \\
\stackrel{2}{0} \\
\stackrel{2}{2} \\
0\end{array}$ & & 点 & 28 & 욤 & n & $\stackrel{10}{7}$ & $\cong$ & $\stackrel{15}{m}$ & In & 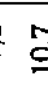 & 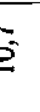 & $\begin{array}{l}\infty \\
0^{-}\end{array}$ & $\stackrel{0}{=}$ & $\stackrel{\infty}{=}$ & $\begin{array}{l}12 \\
\infty\end{array}$ & $\stackrel{0}{\circ}$ \\
\hline 㤩㤩 & & $\begin{array}{l}5 \\
5 \\
5\end{array}$ & 80 & $\begin{array}{l}\tilde{y} \\
\tilde{y}\end{array}$ & 용 & $\stackrel{\text { in }}{\text { I }}$ & $\stackrel{\infty}{=}$ & $\stackrel{10}{=}$ & $\tilde{\infty}$ & $\stackrel{\infty}{\alpha}$ & & $\stackrel{N}{\beth}$ & $\stackrel{10}{0}$ & $\stackrel{0}{8}$ & 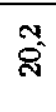 & $\begin{array}{l}\infty \\
\infty \\
\longrightarrow\end{array}$ \\
\hline 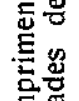 & \multicolumn{2}{|c|}{ 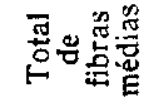 } & 28 & $\hat{\nabla}$ & $\begin{array}{l}y \\
\infty \\
\infty\end{array}$ & 8 & $8^{10}$ & ? & พิ & 1 & 3 & $\begin{array}{l}0 \\
\text { mi }\end{array}$ & $\infty$ & 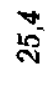 & $\begin{array}{l}\infty \\
10\end{array}$ & 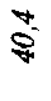 \\
\hline 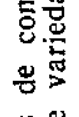 & \multirow{2}{*}{ 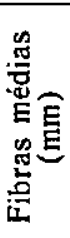 } & $\begin{array}{l}0 \\
\frac{1}{0}\end{array}$ & 20 & ำ & $\stackrel{v}{i v}$ & $\stackrel{2}{0}$ & $\mathrm{E}$ & 용 & $\stackrel{0}{\text { ஸ }}$ & $\frac{10}{5}$ & $\frac{2}{5}$ & $\stackrel{N}{\infty}$ & $\frac{0}{i}$ & $\mathbb{N}^{-1}$ & $\stackrel{\infty}{\mathscr{N}}$ & $\stackrel{N}{=}$ \\
\hline 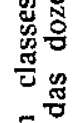 & & $\begin{array}{l}\vec{\varphi} \\
\frac{1}{+}\end{array}$ & 20 & $\stackrel{n}{\sim}$ & $\stackrel{0}{\mathrm{~N}}$ & ָ) & $\stackrel{\infty}{\text { N }}$ & ஜํํ & $m$ & ร & vi & $\stackrel{\infty}{\stackrel{\infty}{m}}$ & $\begin{array}{l}\infty \\
\stackrel{\infty}{N}\end{array}$ & $\begin{array}{l}\sim \\
\text { D }\end{array}$ & 요 & $\stackrel{\sim}{\tilde{N}}$ \\
\hline 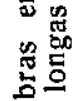 & \multicolumn{2}{|c|}{ 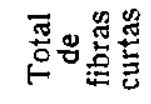 } & 20 & $\stackrel{\infty}{\infty}$ & $\stackrel{v}{\tilde{s}}$ & $\stackrel{m}{\vartheta}$ & $=$ & 10 & $\infty$ & 3 & $\stackrel{n}{v}$ & 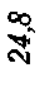 & $\infty^{20}$ & $\infty$ & $\stackrel{v}{\sigma}$ & $\stackrel{+}{\sim}$ \\
\hline $8: \frac{\pi}{3}$ & \multirow{4}{*}{ 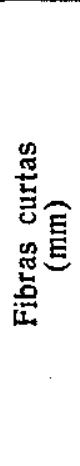 } & 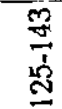 & 80 & $\stackrel{m}{\sigma}$ & $\stackrel{\text { 心 }}{\text { N }}$ & ก & $\underline{0}$ & s- & 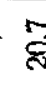 & $\stackrel{s}{a}$ & $\infty^{-}$ & $\stackrel{\infty}{\infty}$ & 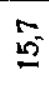 & $\stackrel{N}{N}$ & $\tilde{\sigma}$ & $\stackrel{\infty}{\infty}$ \\
\hline 昰哭 & & $\frac{10}{9}$ & 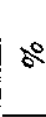 & 2 & $\stackrel{N}{N 1}$ & $\underset{ \pm}{ \pm}$ & $\stackrel{\infty}{0}$ & $\stackrel{\infty}{\mathrm{i}}$ & $\frac{\infty}{\pi}$ & is & is & $\stackrel{\infty}{\infty}$ & $\stackrel{\infty}{\infty}$ & 品 & 1 & $0^{\infty}$ \\
\hline 莒 & & $\frac{5}{8}$ & 50 & I & 1 & 요 & 1 & 1 & \pm & & 1 & $\stackrel{N}{0}$ & 1 & $\stackrel{\infty}{ \pm}$ & 1 & 8 \\
\hline 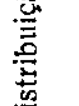 & & 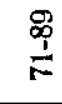 & 20 & 1 & 1 & $\stackrel{\infty}{0^{-}}$ & 1 & 1 & 인 & & 1 & 1 & 1 & $\stackrel{\infty}{=}$ & 1 & $\stackrel{\infty}{0}$ \\
\hline 遂 & & & & 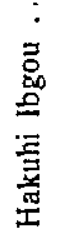 & 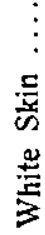 & 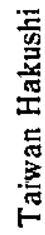 & 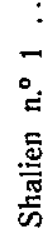 & 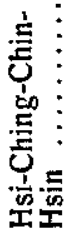 & 苍 & : & 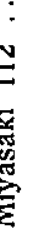 & $\begin{array}{c}\vdots \\
\vdots \\
\frac{1}{5} \\
\text { 山 }\end{array}$ & 莺 & 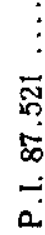 & $\begin{array}{c}\vdots \\
\vdots \\
\bar{\Phi} \\
\frac{D}{00} \\
\overline{0}\end{array}$ & $\begin{array}{l}\vdots \\
- \\
0 \\
\dot{E} \\
\bar{E} \\
\overline{0}\end{array}$ \\
\hline
\end{tabular}



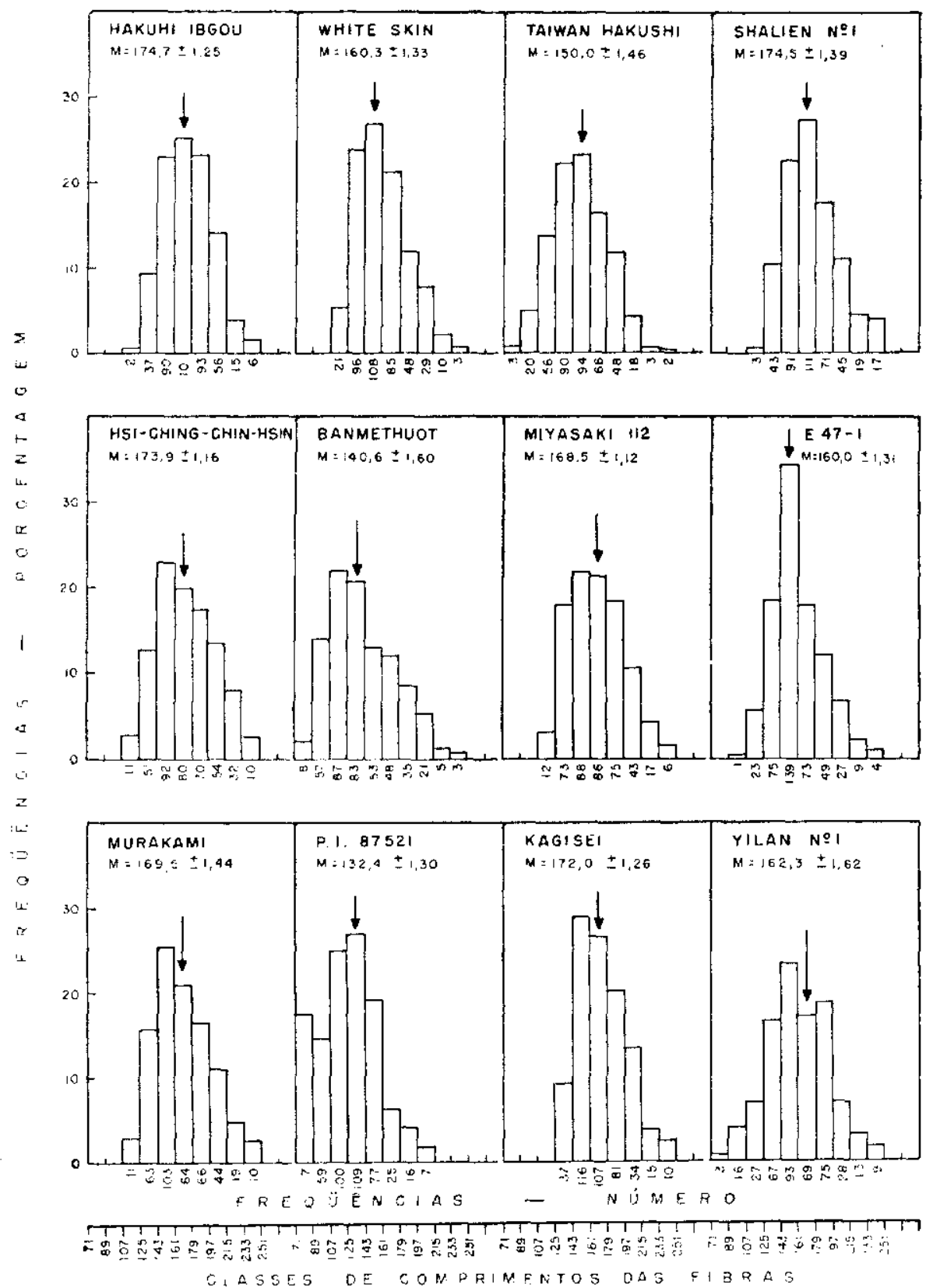

Figlka 1. - Distribuição de 400 fibras, de doze variedades de rami, em classes de comprimento de fibra. 
Sabe-se que as variedades de fibras finas produzem menos fibras por área (11). Como essas fibras são as mais procuradas pelos industriais que fabricam tecidos de natureza mais delicada, cabe aos melhoristas tentar, por meio de cruzamentos específicos, obter plantas que aliem a característica de fibra fina com a de boa produção, levando em conta, também, o seu comprimento e a sua uniformidade.

Os dados obtidos no presente trabalho podem ser usados tanto pelos industriais como pelos melhoristas, seja na obtenção de melhor industrialização da fibra, seja nos trabalhos de melhoramento do rami.

\section{$4-$ CONCLUSÕES}

a) As variedades estudadas diferiram estatisticamente quanto à fineza da fibra. A variedade Taiwan Hakushi possui as fibras mais finas, enquanto $\mathrm{Hsi}-\mathrm{Ching-Chin-Hsin,} \mathrm{as} \mathrm{fibras} \mathrm{mais} \mathrm{grossas.} \mathrm{A} \mathrm{varie-}$ dade Murakami, atualmente a mais cultivada em nosso meio, demonstrou possuir uma das mais grossas fibras, o que explica as dificuldades dos fiandeiros em produzir fios de títulos elevados.

b) As variedades diferiram, ainda, quanto ao comprimento médio de fibra, tendo-se notado uma certa relaçăo direta entre comprimento e uniformidade de comprimento da fibra. As variedades Hakuhi Ibgou, Hsin-Ching-Chin-Hsin e Shalien $n .^{\circ} 1$ apresentaram comprimentos médios de fibras mais altos, aliados a porcentagens mais elevadas de fibras longas. As variedades P.I. 87.521, Banmethuot e Taiwan Hakushi apresentaram os mais baixos comprimentos médios de fibra associados aos mais altos indices de fibras curtas.

\section{FINENESS AND LENGTH OF FIBER OF TWELVE RAMIE VARIETIES}

\section{SUMMAR Y}

A study concerning the fineness and length of the following international varieties of ramie (Boehmeria nivea Gaud.) is reported: Hakuhi lbgou; White Skin; Taiwan Hakushi; Shalien n. $^{\circ}$; Hsi-Ching-Chin-Hsin; Banmethuot; Miyasaki 112; E 47-1; Murakami; P.I. 87.521; Kagisei; and Yilan n. ${ }^{\circ}$.

The study was based on fibers obtained from three month old stalks of the ramie varieties produced in the State of São Paulo, Brazil.

The Taiwan Hakushi exceeded the others varieties in average fineness ( $\mathrm{Nm} \mathrm{1786)}$ and the Hsi-Ching-Chin-Hsin had the lower average fineness ( $\mathrm{Nm}$ 945).

The varieties P.I. 87.521, Banmethuot e Taiwan Hakushi had the lower fiber length (average of $133 \mathrm{~mm}, 141 \mathrm{~mm}$ and $151 \mathrm{~mm}$, respectively). Hakuhi Ibgou, Hsi-Ching-Chin-Hsin and Shalien n. 1 had the longer fibers (average of $175 \mathrm{~mm}$, respectively). 
The fibers classified in short, median and long, showed that the varieties of high average length fiber were more uniform than varieties of low average length fiber.

The results obtained may be used by the ramie industry for production of different kinds of manufactures and also by the breeders in order to establish any breeding and selection program.

\section{LITERATURA CITADA}

1. BEAUVERIE, J. Les textiles végétaux. Paris, Gauthier-Villars, 1913. $730 \mathrm{p}$.

2. CASTRO, G. A. P. CORREIA, F. A. Algumas determinações físicas e químicas da fibra de rami. Bragantia 8:109-117, 1948.

3. CiaRAMEllo, D.; MEDINA, J. C. \& SAlgado, A. L. B. Comprimento e diâmetro dos caules de rami e as características da fibra. Bragantia $22: 73-80,1963$.

4. GRILLOT, G. \& FRANQUIM, P. "La ramie et son amélioration". Cahiers de la Recherche Agronomique, Rabat 4:333-417, 1951.

5. KIRBY, R. H. Vegetable fibers: botany, cultivation and utilization. London, Leonard Hill, 1963. 464p.

6. MATTHEWS, J. M. The textiles fibers. 4th ed. New York, John Wiley, 1924. $411 \mathrm{p}$.

7. MEDINA, J. C.; CIARAMELlo, D.; TOSELO, R. N. \& VENTURINI, W. R. $A$ influência dos fatores irrigação e estação do ano sobre a fineza $e$ a resistência da fibra de rami. Bragantia $20: 841-856,1961$.

8. ROEHRICH, O. \& BUI-XUAN-NHUAN. La fibre de ramie, ses proprietés, ses qualités textiles. Coton Fibr. trop. 1:261-289, 1946.

9. SEALE, C. C. \& ALLISON, R. V. Strenght and fineness of ramie fiber. Proc. Soil Crop Sci. Soc. Flo. 18:300-305, 1958.

10. TONELLI, L. Fibre tessili: filatura. 2ed. Milano, Ulrico Hoepli, 1945. 518p. (Tecnologia tessile, v. 1)

11. VREESWIJK, A. C. W. van. De cultuur winning en verwerking van de ramievezel in Japan. Indonesia, Ministar van Welvaart, 1950. 185 p. (Mededeeling 16) 